\title{
Slipping magnetic reconnection and complex evolution of a flux rope and flare ribbons
}

\author{
Ting Li and Jun Zhang \\ Key Laboratory of Solar Activity, National Astronomical Observatories, Chinese Academy of \\ Sciences, Beijing 100012, China \\ email: liting@nao.cas.cn
}

\begin{abstract}
Abundant observations in recent years show that the flares are more complex than the 2D standard flare model presents. This proposes a challenge to the $2 \mathrm{D}$ flare model and 3D flare model has been developed. We report the complex evolution of flare ribbons and a flux rope in a C8.9 flare event. The two ribbons slipped in opposite directions along the neutral line and the eastern ribbon seemed a hook-like structure. The flare loops were crossed each other, composing a "bi-fan" system. The slipping magnetic reconnection is involved in the flare and leads to slipping motion of flare ribbons and complex evolution of flare loops. Overlying the flare loops, a large-scale flux rope was erupted and meanwhile the eastern end of the flux rope changed with time and slipped along the hook-like ribbon. The fine structures of the flux rope delineated a "triangle-flag" surface, which may imply one-half of the coronal quasi-separatrix layers that surrounds a flux rope. We suggest that the heating process of slipping magnetic reconnection during the flare caused the apparent motion of the flux rope ends.
\end{abstract}

Keywords. Sun: flares, Sun: magnetic fields, Sun: filaments

\section{Introduction}

Solar flares are among the most energetic events of the solar system. Magnetic reconnection during the solar flares releases free magnetic energy that has been stored in the coronal magnetic field. Several observational characteristics are involved in the flares, including flux ropes, flare ribbons and flare loops. Flux ropes were twisted magnetic field lines around an axis, and have been thought to be the origin of coronal mass ejections ( $\mathrm{Li}$ \& Zhang 2013; Patsourakos et al. 2013). The two-dimensional CSHKP model is usually applied to explain the eruptive flares, especially two-ribbon flares (e.g., see the review of Shibata \& Magara 2011). In the CSHKP model, the flux rope is ejected and magnetic reconnection occurs at the null points below the eruptive flux rope. Flare ribbons are formed due to the interaction of the energetic particles and the chromosphere (Schmieder et al. 1987). With the developing of magnetic reconnection at higher altitudes, the two flare ribbons are separated each other and move perpendicular to the neutral line.

However, abundant observations in recent years show that the flares are more complex than the 2D CSHKP model presents. This proposes a challenge to the CSHKP model and thus 3D flare model has been developed (Aulanier et al. 2012; Janvier et al. 2013). Slipping magnetic reconnection belongs to the domain of 3D flare model. A series of magnetic reconnection occurs in the quasi-separatrix layers (QSLs; Priest \& Démoulin 1995; Démoulin et al. 1996) and exchanges the connectivity of magnetic fields with neighboring field lines (Aulanier et al. 2012; Li \& Zhang 2014). During this process, the flare loops display the apparent slipping motion along the QSLs. The slipping motion of flare loops and ribbons are deemed as the occurrence of slipping magnetic reconnection. 


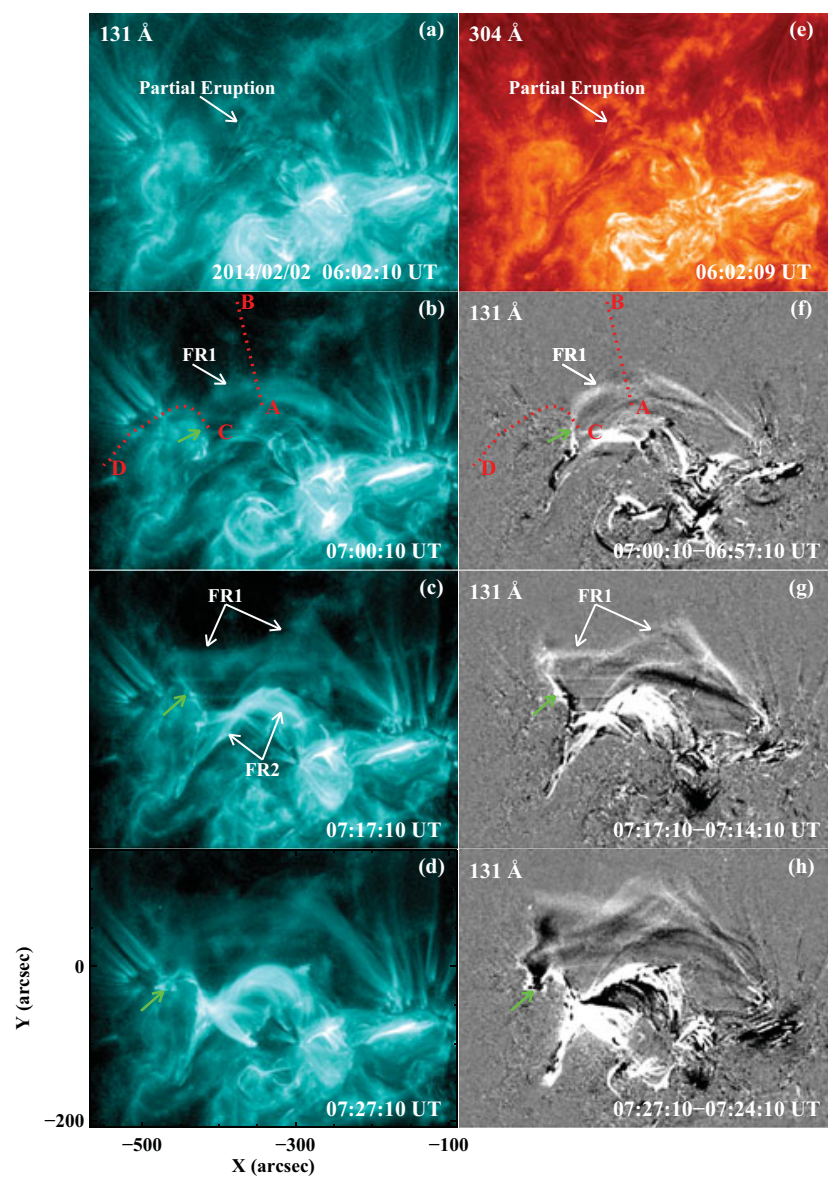

Figure 1. Panels (a)-(d): SDO/AIA $131 \AA$ images showing the flare and the erupting flux rope (FR1). Panel (e): SDO/AIA $304 \AA$ image presenting the partial eruption of the filament. Panels (f)-(h): AIA $131 \AA$ A running-difference images showing the evolution of fine structures of the flux rope. Green arrows point to the eastern footpoints of the flux rope.

\section{Observations and Data Analysis}

The EUV observations of Atmospheric Imaging Assembly (AIA; Lemen et al. 2012) onboard the Solar Dynamics Observatory (SDO; Pesnell et al. 2012) on 2014 February 02 are used to analyze the event. The $S D O /$ AIA uninterruptedly observes the full disk of the Sun in 10 (E)UV channels at a spatial resolution of 1.5 arcsec and $12 \mathrm{~s}$ cadence. A C8.9 flare occurred in NOAA AR 11967, including two negative-polarity flare ribbons (NR1 and NR2 in Figure 2) and one positive-polarity ribbon (PR in Figure 2).

\section{Results}

Starting from 05:30 UT, the filament material was activated and moved from its west end towards the east end (Figures 1(a) and (e)). The C8.9 flare initiated at 07:17 UT, and the overlying large-scale flux rope was also erupted. An interesting phenomenon is that the eastern footpoints of the flux rope moved along the flare ribbon while its western end remained fixed (Figure 1). The flux rope seemed to be composed of a bundle of loop-like 


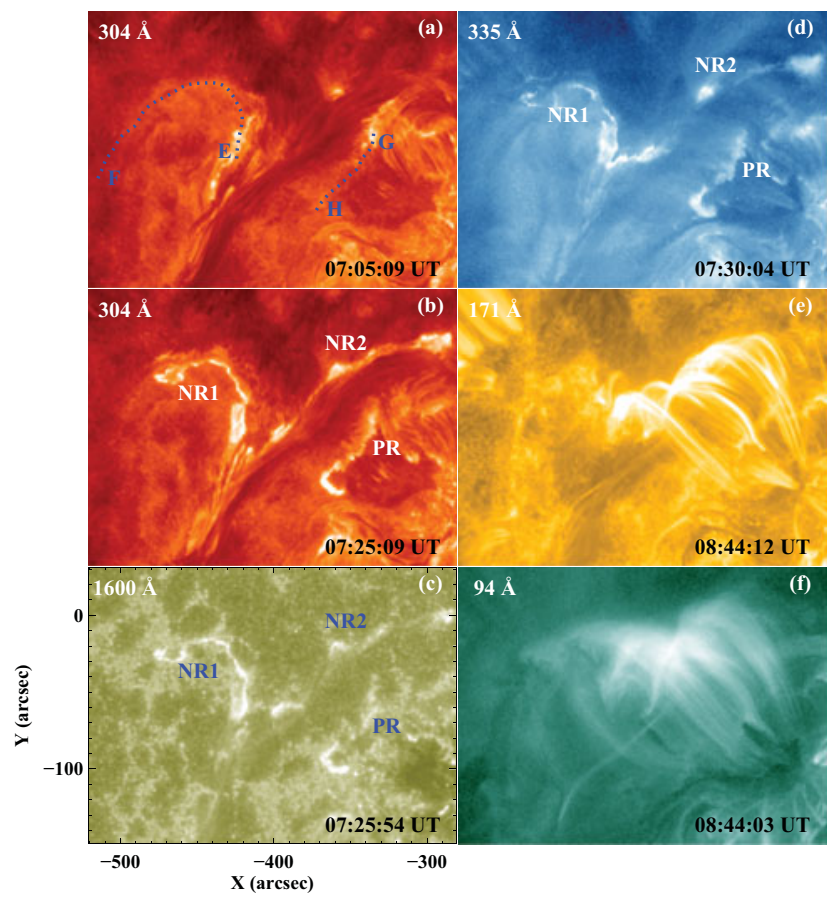

Figure 2. $S D O /$ AIA multi-wavelength images showing the evolution of flare ribbons and post-flare loops. NR1 and NR2 are two negative-polarity ribbons and PR is one positive-polarity ribbon.

fine structures, which delineated a "triangle-flag" topology. It could only be observed in high-temperature wavelengths, such as $131 \AA$ and $94 \AA$.

Red dotted lines "A-B" and "C-D" show the positions of the cuts used to obtain the stack plots shown in Figures 3(b) and (c). The initial rise velocity of the fine structures of the FR1 is about $20 \mathrm{~km} \mathrm{~s}^{-1}$. At about 07:24 UT, the fine structures rose upward rapidly with a velocity of $130 \mathrm{~km} \mathrm{~s}^{-1}$. The propagation velocity of the eastern end of FR1 was about $60 \mathrm{~km} \mathrm{~s}^{-1}$. Below the large-scale flux rope, flare loops appeared. The evolution of flare loops was very complex, and they were crossed and intersected each other. The eastern ends of the successively visible flare loops propagated towards the north and the western ends of flare loops propagated in the opposite direction. At last, they composed a "bi-fan" loop system.

Three flare ribbons are involved in the flare, including NR1, NR2 and PR (Figure 2). The appearance of post-flare loops showed that partial flare loops connected PR and NR2, and south flare loops connected PR and NR1. The propagation of ribbon NR2 was not obvious, while the ribbons PR and NR1 slipped in opposite directions during the process of flare evolution. The eastern end of eruptive flux rope was at the leading edge of the ribbon NR1. The ribbon NR1 finally formed a hook-like structure. The NR1 and NR2 lie at the negative-polarity region that corresponds to the faculae of the AR. The $\mathrm{PR}$ is located in positive-polarity fields nearby a sunspot of the AR. Blue dotted curves "E-F" and "G-H" show the positions of the cuts used to obtain the stack plots shown in Figures 3(d) and (e). The NR1 lasts about $40 \mathrm{~min}$ and the average velocity of the NR1 is approximately $50 \mathrm{~km} \mathrm{~s}^{-1}$, similar to the propagation velocity of the eastern ends of the overlying flux rope. The $\mathrm{PR}$ has a length of about $40 \mathrm{Mm}$, and propagates to the south with a velocity of $30 \mathrm{~km} \mathrm{~s}^{-1}$. 

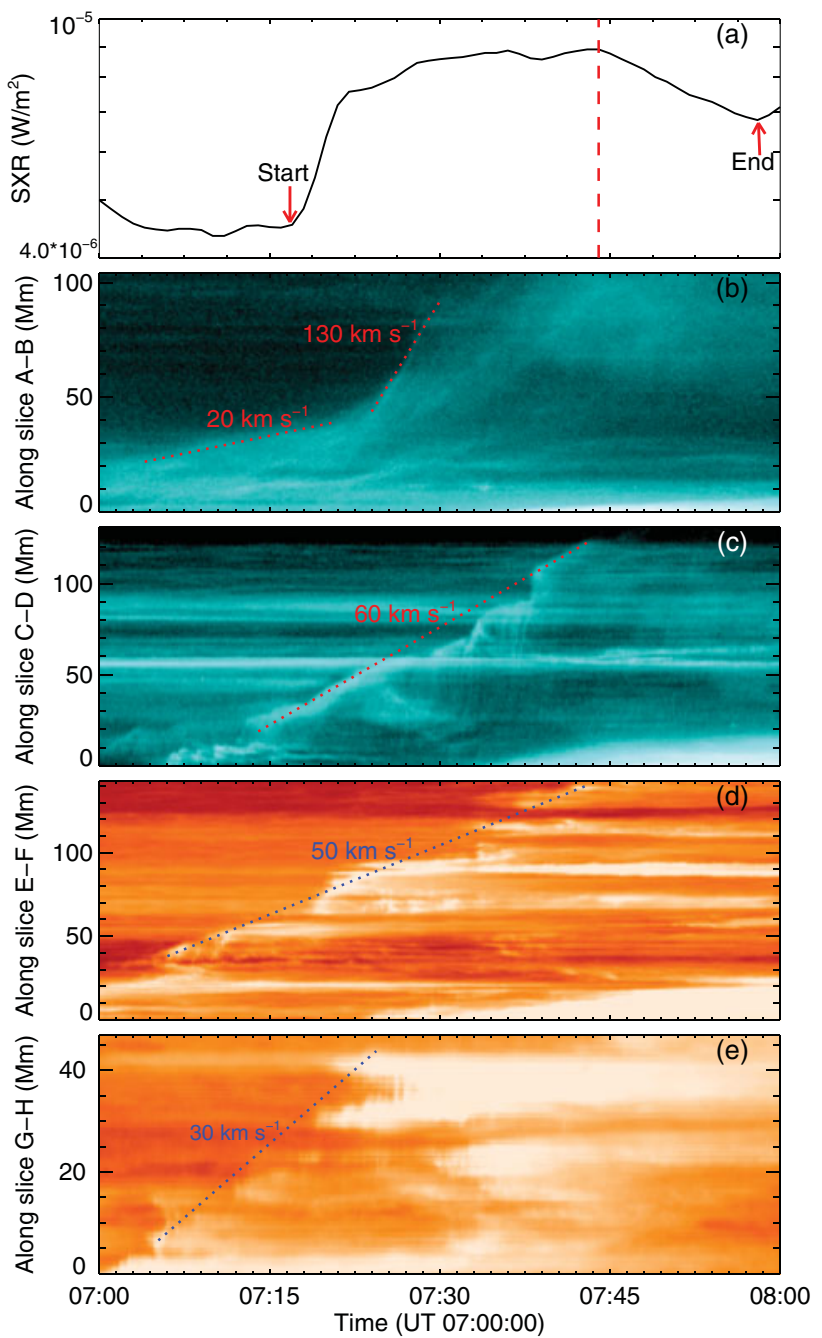

Figure 3. Panel (a): GOES SXR 1-8 A flux of the associated flare. Red dashed line denotes the flare peak time. Panels (b)-(c): stack plots along slices "A-B" and "C-D" (red dotted lines in Figure 1(b)) at $131 \AA$ showing the eruption of the flux rope FR1 and the propagation of its eastern end. Panels (d) $-(\mathrm{e})$ : stack plots along slices " $\mathrm{E}-\mathrm{F}$ " and " $\mathrm{G}-\mathrm{H}$ " (blue dotted lines in Figure 2(a)) at $304 \AA$ showing the propagations of flare ribbons NR1 and PR.

The ribbons NR2 and PR are at the both sides of the filament. GOES soft X-ray 1-8 $\AA$ flux showed that the C8.9 flare reached its peak at 07:44 UT and ended at 07:58 UT (Figure 3(a)). At the late phase of the flare, the flare loops connecting NR2 and PR could still be observed. The post-flare loops observed at $94 \AA$ were extended to a higher altitude than the loops observed at $171 \AA$ (Figures $2(\mathrm{e})$ and (f)). This may imply that the high-temperature loops are located outside the low-temperature loops.

\section{Summary and Discussion}

Our observations present the unique and extreme rare event, which could be explained by the 3D flare model (Janvier et al. 2015). According to the 2D CSHKP model, the two flare ribbons are roughly parallel to the neutral line and exhibit a separate motion 
away from the neutral line with the occurrence of magnetic reconnection of higher magnetic fields (Asai et al. 2004; Reid et al. 2012). The flare loops connecting two ribbons are approximately potential fields and parallel to each other. In our observations, the topologies of flare ribbons and loops are more complex than expected. The eastern flare ribbon seemed a hook-like structure and both ribbons slipped in opposite directions. The separation motion away from the neutral line was not obvious. The flare loops are crossed each other, composing a "bi-fan" system. We suggest that slipping magnetic reconnection is involved in the flare and leads to slipping motion of flare ribbons and complex evolution of flare loops.

Moreover, previous observations of eruptive flux ropes give us an image that the ends of the flux ropes could not move during the eruption process (Zhang et al. 2015). In this event, the eastern end of the flux rope changed with time and slipped along the flare ribbon. There are two possibilities that may account for the evolution. One possibility is that the slipping magnetic reconnection during the flare heats the ends of the flux rope and causes the successive visibility of fine-structures of the flux rope. In this situation, the "triangle-flag" magnetic fields exist before their appearance and become visible due to the heating process of the flare. Another possibility involves the reconnection of the fluxrope field lines with ambient magnetic field lines. The successive reconfiguration of fluxrope field lines during the process of slipping reconnection induces the apparent slipping motion of the footpoints of the flux rope. However, the evidence for the reconnection of the flux rope is not observed.

The slipping velocity is about $50-60 \mathrm{~km} \mathrm{~s}^{-1}$, similar to that of the slipping flare loops in Dudík et al. (2014). Recent observations of Li \& Zhang (2015) showed that the slipping flare loops and the small-scale substructures within the flare ribbon both exhibited quasi-periodic oscillations with periods of 3-6 min, which are attributed to quasi-periodic slipping reconnection. The whole scenario of the event well matches the $3 \mathrm{D}$ numerical simulations by Titov (2007) and Janvier et al. (2013). In their simulations, the hyperbolic flux tube is located below the flux rope and the photospheric footprints of the hyperbolic flux tube correspond to the location of hook-shaped structure of the flare ribbon. The "triangle-flag" surface in our event may imply one-half of the coronal QSL that surrounds a flux rope. The 3D flare models have been developed in recent years, however, the observations about the 3D magnetic reconnection, especially the slipping reconnection are still not rich. With the increasing time and spatial resolution, more observational studies will be carried out and meanwhile promote the theoretical development.

\section{Acknowledgements}

We acknowledge the $S D O$ for providing data. This work is supported by the National Natural Science Foundations of China (11303050, 11533008 and 1221063) and the Strategic Priority Research Program-The Emergence of Cosmological Structures of the Chinese Academy of Sciences, Grant No. XDB09000000.

\section{References}

Asai, A., Yokoyama, T., \& Shimojo, M., et al. 2004, ApJ, 611, 557

Aulanier, G., Janvier, M., \& Schmieder, B. 2012, A\&A, 543, A110

Démoulin, P., Henoux, J. C., Priest, E. R., \& Mandrini, C. H. 1996, A\&A, 308, 643

Dudík, J., Janvier, M., \& Aulanier, G., et al. 2014, ApJ, 784, 144

Janvier, M., Aulanier, G., Pariat, E., \& Démoulin, P. 2013, A\&A, 555, A77

Janvier, M., Aulanier, G., \& Démoulin, P. 2015, Sol. Phys., 63

Li, T. \& Zhang, J. 2013, ApJL, 778, L29 
Li, T. \& Zhang, J. 2014, ApJL, 791, L13

Li, T. \& Zhang, J. 2015, ApJL, 804, L8

Lemen, J. R., Title, A. M., \& Akin, D. J., et al. 2012, Sol. Phys., 275, 17

Patsourakos, S., Vourlidas, A., \& Stenborg, G. 2013, ApJ, 764, 125

Pesnell, W. D., Thompson, B. J., \& Chamberlin, P. C. 2012, Sol. Phys., 275, 3

Priest, E. R. \& Démoulin, P. 1995, J. Geophys. Res., 100, 23443

Reid, H. A. S., Vilmer, N., Aulanier, G., \& Pariat, E. 2012, A\&A, 547, A52

Schmieder, B., Forbes, T. G., Malherbe, J. M., \& Machado, M. E. 1987, ApJ, 317, 956

Shibata, K. \& Magara, T. 2011, Living Reviews in SolarPhysics, 8, 6

Titov, V. S. 2007, ApJ, 660, 863

Zhang, J., Yang, S. H., \& Li, T. 2015, A\&A, 580, A2 